\title{
A Model of the Human Eye
}

\author{
G. Colicchia, H. Wiesner, and C. Waltner, Ludwig Maximilian University, Germany \\ D. ZollIman, Kansas State University, Manhattan, KS
}

W e describe a model of the human eye that incorporates a variable converging lens.

The model can be easily constructed by students with low-cost materials. It shows in a comprehensible way the functionality of the eye's optical system. Images of near and far objects can be focused. Also, the defects of near and farsighted eyes can be demonstrated.

A commercial product is available for demonstrating the optics of the eye. ${ }^{1}$ This model is based on the Ingersoll Eye Model, which was sold by Cenco and is apparently no longer available. However, this model does not have the variable lens capability. One with a variable lens is quite expensive. ${ }^{2}$

\section{Functionality of the Eye}

The task of the eye's optical system is to create an optimal sharp image of the external world on the retina. ${ }^{3}$ With a normal eye, rays from objects at many different distances pass through the lenses and fluids and are focused on the retina. Defects in the eye's optical system can lead to many different difficulties in seeing. Here we consider the normal-sighted eye and two common problems - nearsighted and farsighted eyes.

a) Normal-sighted eye. The optical system of the eye focuses all parallel rays coming from a distant point object at a quasi point $\mathrm{P}^{\prime}$ on the retina [Fig. $1(a)$. When the eye is looking at a near point $P$, an increase in the curvature of the crystalline lens focuses divergent rays coming from that point. Like the point $\mathrm{P}$, all other points of the object are focused at separate points of the retina, where they form a sharp image of the object. b) Nearsighted eye (myopia). The optical system of the nearsighted eye has a refractive power that is too large relative to the axial length. The focused image from a distant object will be formed in front of the retina. Thus, the image that is formed on the retina will be out of focus. However, if the object is near to the eye, the image on the retina will be in focus. Fig. $1\left(b_{1}\right)$ shows axial myopia, in which the refractive power of the crystalline lens is normal but the eyeball is too long. Fig. $1\left(b_{2}\right)$ shows refractive myopia, in which the length of the eyeball is normal but the refractive power of the lens is too strong.

c) Farsighted eye (byperopia). The optical system of the farsighted eye has a refractive power that is too weak relative to the axial length of the eye. Therefore, the relaxed hyperopic eye focuses the image of distant objects behind the retina, resulting in a blurred image on the retina. Looking at closer objects makes the vision worse by moving the image even farther behind the retina.

In younger people, the crystalline lens can often accommodate enough to overcome the need for more refractive power. This accommodation results in clear images, at least for distant objects. As the hyperopic eye ages, accommodation initially fails for near objects, and later for distant objects.

Fig. 1( $\left.c_{1}\right)$ shows axial hyperopia, in which the opti$\mathrm{cal}$ system of the eye is normal but the eyeball is too short. Fig. $1\left(c_{2}\right)$ shows refractive hyperopia, in which the eyeball is normal in length but the optical system of the eye has insufficient converging power.

As shown in Fig. 1, the lens of the eye must change 


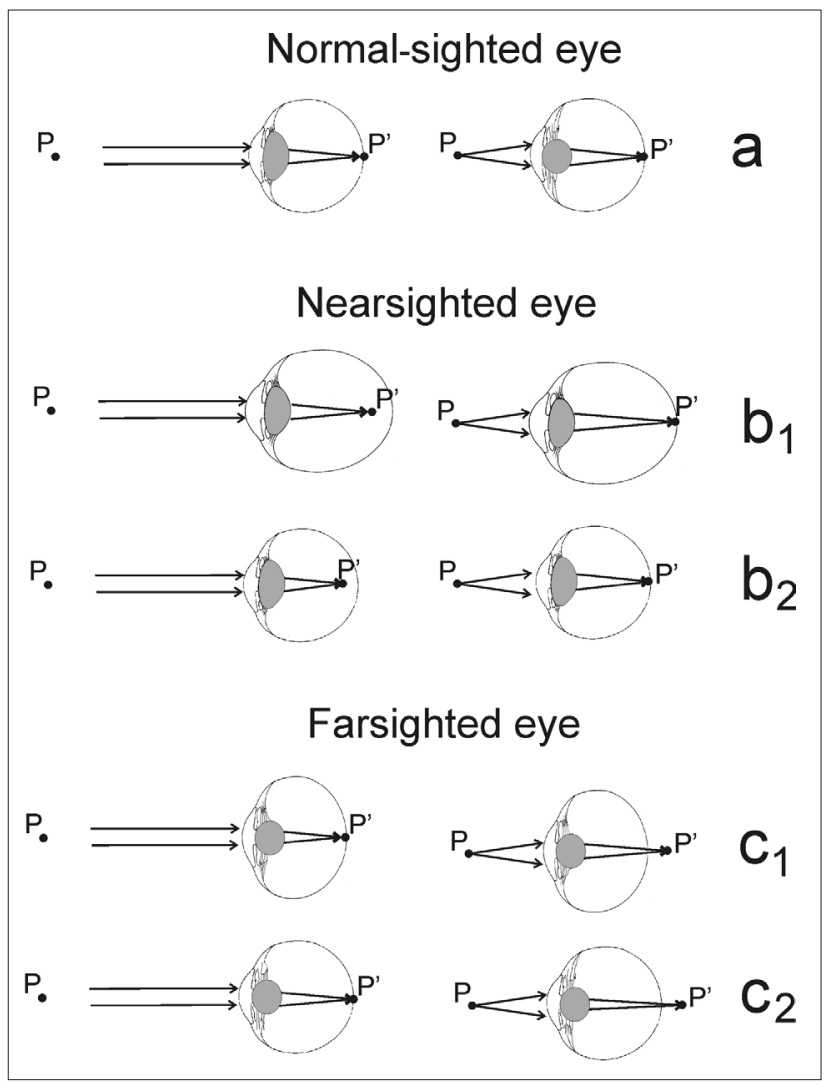

Fig. 1. Focusing in normal and defective eyes for distant (left) and near (right) objects.

its shape so that we can see objects at different distances. The change in shape changes the refractive power of the lens and allows us to focus the images of objects located at vastly different distances on the retina. In our eyes, the changes in the lenses are caused by contraction and relaxation of the ciliary muscles. The process is called accommodation.

\section{Construction of the Eye Model}

Our eye model consists of a hollow polystyrene ball (diameter $\approx 15 \mathrm{~cm}$ ) that represents the ocular globe (Fig. 2). A hole in the sphere, about $2 \mathrm{~cm}$ in diameter, is covered with a glass or plastic lens $\mathrm{C}(f=18 \mathrm{~cm})$, which simulates the optical refractive power of the cornea. On the opposite surface of the ball a segment, about $6 \mathrm{~cm}$ in diameter, is cut out and covered with transluscent paper $\mathrm{R}$. This represents a portion of the retina. Inside the sphere, a lens with a variable focal length ${ }^{4}$ is arranged in the optical path between the "cornea" C and the semitransparent surface $\mathrm{R}$.

The variable lens (Fig. 3) consists of a transparent
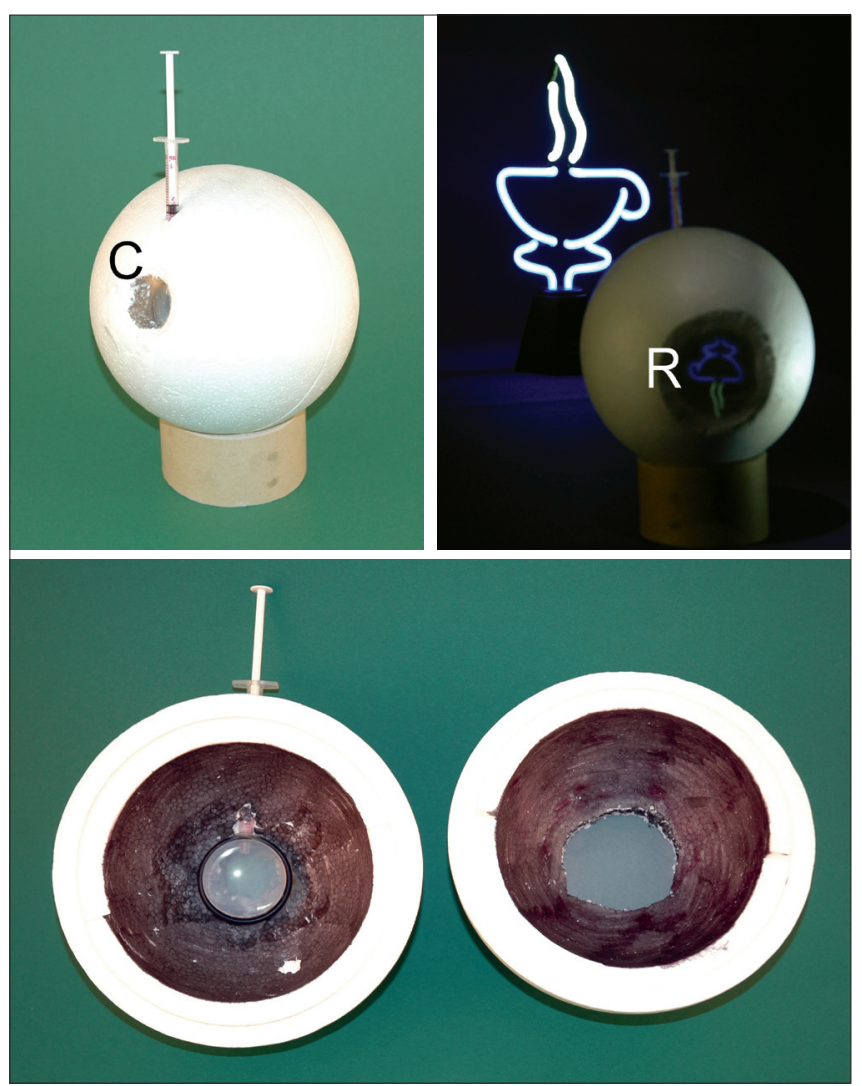

Fig. 2. Eye model (above) from the front and from behind (below); the inside is painted black in order to decrease reflected light.

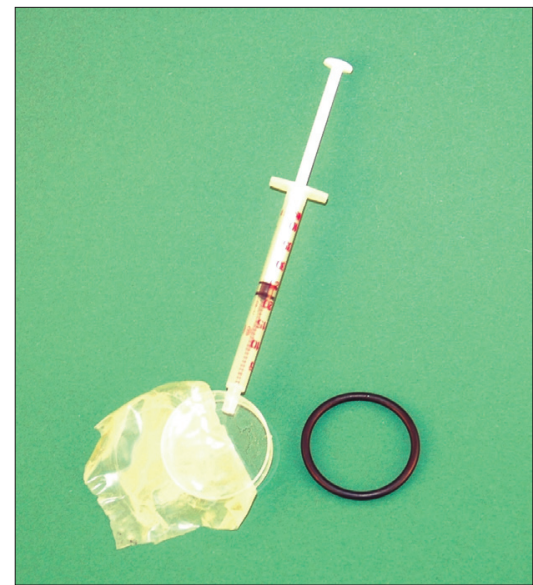

Fig. 3. Elements of the water-filled lens.

plastic petri dish (diameter $3.5 \mathrm{~cm}$ ) that is filled with water and covered with a transparent film. The film needs to be elastic and moderately strong so that it will withstand repeated use by students. We have found that a small segment cut from a transparent condom is very effective for this purpose. 


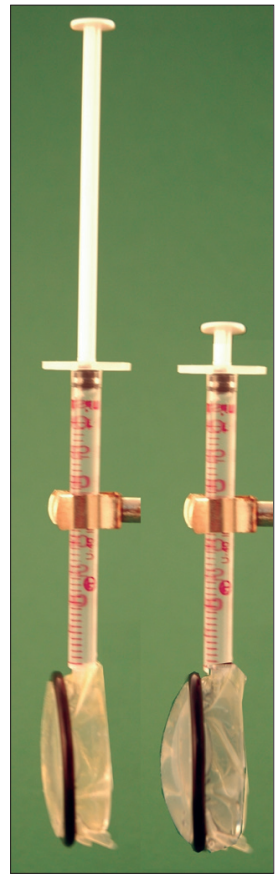

The elastic film is stretched on the open side of the petri dish and fixed by a rubber band or O-ring. To insert the water, a small hole is created in the side of the dish. Using a small soldering iron to melt the hole is a simple way to accomplish this. When the lens is filled with water from a syringe, the elastic film is deformed into a convex shape. By varying the water volume in the lens, the curvature of the elastic film changes (Fig. 4). Thus, the refractive power of the liquid lens can be varied continuously.

The changing refractive power of the liquid model lens represents the accommodation of the eye's crystalline lens. When water is added to the model lens, its variable surface becomes more convex. This process is similar to increasing the power of the eye's crystalline lens to focus on closer objects.

\section{Images of Bright Objects}

To focus an image on the retina $R$, the refractive power of the lens of the eye model needs to match, in a sense, the axial length of the model (or vice versa). This is accomplished by adding the refractive powers of the liquid lens and lens C. Objects from $\infty$ to $50 \mathrm{~cm}$ can be put in focus by adding or removing approximately $1 \mathrm{ml}$ of water with the syringe.

Because of the restricted refractive power of the

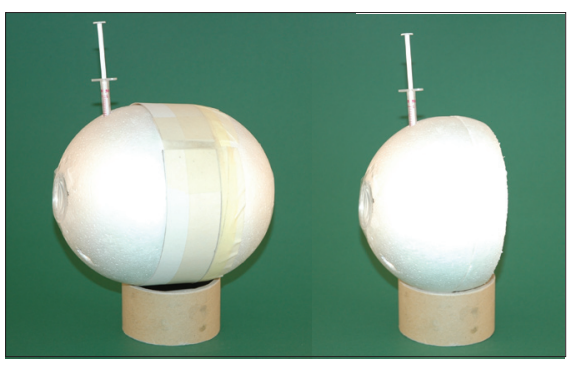

Fig. 5. A longer model simulating myopia and shorter model simulating hyperopia.

liquid lens, defective vision can be simulated by changing the axial length of the model. Myopia is simulated by elongating and hyperopia by shortening of the axis of a ball (Fig. 5). The same defective vision can be simulated by a normal length ball with the lens $\mathrm{C}$ having too strong $(f=10.6 \mathrm{~cm})$ or too weak $(f=22.5 \mathrm{~cm})$ refractive power.

By placing ordinary spectacles in front of the models, the refractive sight defects can be corrected. In models with a pinhole aperture, all focus defects become less noticeable in bright light. ${ }^{5}$ This effect is similar to an eye with the pupil closing to a pinhole in which the depth of field will be enhanced, and the blurredness of image is reduced without improving focusing.

\section{The Optics Simulation Program}

The eye models show very well the change of imaging by modification of refractive power. However, the light paths are not visible. Therefore we recommend the freely available simulation program "Optics," which was developed for the project Modern Miracle Medical Machines in cooperation with Professor Dean Zollman (Kansas State University). This program, a lesson that uses the simulation in conjunction with the eye model described here and lists sources for the components of the eye model, is available from http://web. phys.ksu.edu/mmmm or www.didaktik.physik.unimuenchen.de.

This model, in combination with the program, helps students improve their understanding of imaging with a lens as well as the function of lenses in the human eye, including the defective vision and its correction. 


\section{References}

1. PASCO Human Eye Model, Catalog No. 84777, http://store.pasco.com/pascostore/showdetl. cfm? \&DID=9\&Product_ID=56044\&Detail $=1$.

2. Available from several sources. See, for example, American 3B Scientific, https://www.a3bs.com/shop/u.s.a./ eye_models/functional_eye_w16002,p_3_65_30_0_ 4129_searchresult_1_image_movie.html.

3. A. Maheshwari and D.R.Williams, Learning Optics Using Vision; http://textonly.cfao.ucolick.org/EO/ Resources/Learn_optics_AO.pdf.

4. N. Sogiura and S. Morita, "Variable-focus liquid-filled optical lens," Appl. Opt. 32, 4181-4186 (1993).

5. G. Colicchia et al., "Pinhole glasses," Phys. Teach. 46, 26 (Jan. 2008).

PACS codes: 01.50.My, 42.00.00

G. Colicchia, H. Wiesner, and C. Waltner Physics Education, Ludwig Maximilian University, Germany; pino@Irz.uni-muenchen.de hartmut.wiesner@physik.uni-muenchen.de christine.waltner@physik.uni-muenchen.de

D. Zollman, Physics Education, Kansas State University; dzollman@phys.ksu.edu 